\title{
Aptidão física e nível habitual de atividade física associados à saúde cardiovascular em adultos e idosos
}

\section{Physical fitness and habitual level of physical activity associated with cardiovascular health in adults and elderly}

\author{
Átila A. Trapé1; Elisangela A. S. S. Lizzi², André M. Jacomini ${ }^{3}$, Sara C. Hott ${ }^{4}$, Carlos R. Bueno Júnior \\ Anderson S. Zago ${ }^{6}$
}

\begin{abstract}
RESUMO
Modelo do Estudo: Transversal

Fundamento: $O$ nível habitual de atividade física (NHAF) vem sendo utilizado para estabelecer uma relação entre estilo de vida ativo e saúde cardiovascular. No entanto, a avaliação da aptidão física permitiria a avaliação real das condições físicas do indivíduo, podendo assim apresentar melhores relações com a saúde cardiovascular. Objetivos: Relacionar o NHAF e aptidão física com a saúde cardiovascular de adultos e idosos e verificar a associação da prática regular de exercícios físicos supervisionada com os diferentes níveis de aptidão física. Método: Foram avaliados 213 adultos e idosos (> 50 anos) participantes de projetos comunitários. Os participantes realizaram as seguintes avaliações: NHAF por meio do questionário IPAQ, aptidão física por meio de uma bateria de testes motores que permitiu o cálculo do Índice de Aptidão Funcional Geral (IAFG), pressão arterial, perfil lipídico e índice de massa corporal. Resultados: O modelo linear generalizado evidenciou um maior número de diferenças com relação às variáveis relacionadas à saúde cardiovascular quando os participantes foram subdivididos em grupos tendo o IAFG como variável independente, comparado ao NHAF. O teste exato de Fisher evidenciou que os grupos de IAFG classificados como "bom" e "muito bom" apresentaram maior proporção de indivíduos com prática regular de exercícios físicos superior a 6 meses, com destaque para um maior número realizando a prática com supervisão $(p<0,0001)$. Conclusão: O IAFG apresenta melhor associação com a saúde cardiovascular do que o NHAF e o tempo de prática e a supervisão estão associados ao nível de aptidão física.
\end{abstract}

Palavras-chave: Aptidão Física. Atividade Motora. Doenças Cardiovasculares. Envelhecimento. Exercício.

1. Docente. Curso de Educação Física da Universidade Paulista (UNIP), Campus Ribeirão Preto. Professor de Educação Física. Centro de Educação Física, Esportes e Recreação (CEFER) da Universidade de São Paulo (USP) Campus Ribeirão Preto.

2. Docente. Universidade Tecnológica Federal do Paraná.

3. Bacharel em Educação Física, Mestre em Reabilitação e Desempenho Funcional, Faculdade de Medicina de Ribeirão Preto (FMRP) da USP.

4. Farmacêutica, Doutora em Farmacologia, FMRP-USP.

5. Docente. Escola de Educação Física e Esportes de Ribeirão Preto (EEFERP) da USP.

6. Docente. Departamento de Educação Física. Faculdade de Ciências da UNESP, Campus Bauru.
Correspondencia

Prof. Átila Alexandre Trapé Prefeitura do Campus USP de Ribeirão Preto Centro de Educação Física, Esportes e Recreação (CEFER)

Av. Bandeirantes, 3900 CEP 14040-900 - Ribeirão Preto/SP

Artigo recebido em 08/08/2014 Aprovado para publicação em 24/02/2015 


\begin{abstract}
Study design: cross-sectional

Background: The habitual level of physical activity (HLPA) has been used to establish the relation between active lifestyle and cardiovascular health. However, the assessment of physical fitness would review the actual physical condition of the individual, and thus can have better relations with cardiovascular health. Objectives: Relate HLPA and physical fitness with cardiovascular health of adults and elderly and to verify the association of supervised regular physical exercise with different levels of fitness. Method: 213 adults and elderly (> 50 years) participating in community projects were evaluated. The HLPA were assessed by the IPAQ and physical fitness through a battery of motor tests that allowed the calculation of the General Functional Fitness Index (GFFI). Blood pressure, lipid profile and body mass index were considered as indicators of cardiovascular health. Results: The generalized linear model showed a greater number of differences with respect to variables related to cardiovascular health when participants were subdivided having GFFI as an independent variable, compared to HLPA. The Fisher exact test showed that the groups GFFI classified as "good" and "very good" showed a higher proportion of individuals with regular practice for more than 6 months exercise, especially for a larger number performing the practice under supervision $(p<0.0001)$. Conclusion: The GFFI has better association with cardiovascular health than HLPA and time of practice and supervision are associated with the level of physical fitness.
\end{abstract}

Keywords: Physical Fitness. Motor Activity. Cardiovascular Diseases. Aging. Exercise.

\section{Introdução}

Dentre as alterações relacionadas ao processo de envelhecimento e que podem comprometer a qualidade de vida do idoso pode-se destacar a diminuição da aptidão física e a maior vulnerabilidade para o surgimento de doenças crônicas, em especial as doenças cardiovasculares (DCVs). ${ }^{1,2,3}$

A aptidão física envolve um conjunto de atributos relacionados à agilidade, equilíbrio, composição corporal, resistência aeróbica, coordenação, flexibilidade, força e velocidade ${ }^{4}$, e possui relação direta com a capacidade de desempenhar atividades da vida diária de forma segura e eficaz. ${ }^{3,5}$ As DCVs são consideradas as doenças de maior prevalência e principal causa de morte (30 a 40\%) na população adulta e idosa ${ }^{6,7}$, sendo a hipertensão arterial sistêmica (HAS), a obesidade, as dislipidemias e o sedentarismo os principais fatores de risco modificáveis para o desenvolvimento dessas doenças. ${ }^{1,2,6}$

Estudos sugerem que essas alterações podem ser evitadas/amenizadas com a adoção de um estilo de vida ativo. ${ }^{5,6}$ Os exercícios físicos, definidos como atividades físicas planejadas e estruturadas com objetivo de manter/aumentar a saúde e a aptidão física ${ }^{4}$, são utilizados para a prevenção/controle dos fatores de risco das DCVs. ${ }^{5-8}$

Diversos estudos têm objetivado discutir tais relações, entretanto, a maioria deles relacionam o estilo de vida ativo e DCVs por meio de questionários sobre o nível habitual de atividade física (NHAF), que está relacionado a movimentos corporais gerais ${ }^{4}$, e com respostas subjetivas dos participantes por meio da recordação das atividades realizadas na última semana ${ }^{9}$. Vale ressaltar que grande parte destes estu$\operatorname{dos}^{10-12}$ não obtiveram resultados positivos nos parâmetros de saúde. Desta forma, há necessidade de estudos que estabeleçam relação mais concreta entre estilo de vida ativo e saúde cardiovascular, tendo como referência as definições de exercício físico e aptidão física. Os testes motores permitem a avaliação da real condição de aptidão física do indivíduo, que poderá estar melhor relacionada com as variáveis de saúde e do processo de envelhecimento. ${ }^{5,13}$

Diante deste contexto, o objetivo deste estudo foi comparar a relação do NHAF e da aptidão física com a saúde cardiovascular de adultos e idosos e verificar a associação de diferentes níveis de aptidão física com a prática regular de exercícios físicos supervisionados por um profissional de educação física.

\section{Método}

Neste estudo transversal, realizado em Ribeirão Preto/SP, Brasil, foram avaliados 213 adultos com idade superior a 50 anos e idosos, participantes de projetos comunitários. $\mathrm{O}$ cálculo amostral foi baseado nos testes estatísticos realizados para a análise dos dados do presente estudo, considerando o nível de significância de $5 \%$ e poder de $80 \%$. Os critérios de 
inclusão utilizados foram: ser participante regular de projetos comunitários e não apresentar nenhuma condição de saúde que impedisse a realização dos testes motores.

A amostragem foi por conveniência obtida em dois estágios:

- estágio I: seleção de conglomerados que constituem e refletem as características da população, sendo heterogêneos quanto aos aspectos investigados. Tais conglomerados se referem a dois projetos comunitários vinculados às associações de aposentados independentes, dois projetos vinculados às Universidades e um à Prefeitura de Ribeirão Preto/ SP. Tais grupos realizam atividades socioculturais variadas, como artesanato, coral e culinária e/ou prática de exercícios físicos;

- estágio II: convite aos participantes de cada conglomerado, com chances iguais de participação. Durante o processo de recrutamento, observou-se e controlou-se a estratificação dos participantes quanto à idade, escolaridade, renda per capita, cor da pele e NHAF, para melhor caraterização da amostra. ${ }^{14}$

Para avaliar o NHAF dos participantes utilizouse do International Physical Activity Questionnaire (IPAQ), instrumento já validado no Brasil ${ }^{15}$. A coleta dos dados foi realizada por meio de entrevista pessoal na qual avaliou-se a frequência, em dias, e a duração, em minutos, das atividades realizadas por mais de dez minutos contínuos durante uma semana normal. Neste trabalho, utilizou-se a versão curta do questionário, que inclui os quatro componentes da atividade física: atividade física no trabalho, como meio de transporte, em casa e no lazer, classificadas em vigorosas, moderadas e caminhada, segundo o relato dos participantes. A classificação do NHAF foi obtida somando-se a frequência e duração de todas as atividades.

A aptidão física foi avaliada pela bateria de testes motores da American Alliance for Health, Physical Education, Recreation and Dance (AAHPERD), que está diretamente relacionada às atividades cotidianas ${ }^{13,16}$ sendo composta por cinco testes motores envolvendo coordenação, agilidade e equilíbrio dinâmico, flexibilidade, resistência aeróbia e resistência de força ${ }^{13}$. O resultado de cada teste foi classificado de acordo com as tabelas de valores normativos descritas previamente ${ }^{13,17,18}$, variando a pontuação (escore percentil) de cada teste de 0 a 100 . A pontuação de cada teste foi somada obtendo-se o Índice de Apti- dão Funcional Geral (IAFG) individual, que variou de 0 a 500 .

Em relação às variáveis relacionadas aos fatores de risco para DCVs, a pressão arterial (PA) foi avaliada conforme recomendações da VI Diretrizes Brasileiras de Hipertensão ${ }^{7}$, por meio do método auscultatório, utilizando-se um esfigmomanômetro aneroide e estetoscópio da marca Welch Allyn Tycos $D S-44$. Os participantes com valores de PAS e PAD acima de $160 \mathrm{mmHg}$ e $100 \mathrm{mmHg}$, respectivamente, além de não serem inseridos no estudo, foram orientados a procurar serviço médico especializado.

O índice de massa corporal (IMC), massa corporal em quilogramas dividido pela estatura em metros ao quadrado $\left(\mathrm{kg} / \mathrm{m}^{2}\right)$, foi utilizado como indicador de estado nutricional recomendado pela Organização Mundial da Saúde. ${ }^{19}$ A balança e o estadiômetro utilizados foram os da marca Filizola, modelo 31, com resolução de $0,1 \mathrm{~kg}$ e $0,01 \mathrm{~m}$, respectivamente.

Para a coleta e análise da bioquímica sanguínea: (a) os participantes estavam em 12 horas de jejum; (b) o sangue foi analisado em laboratório pelo aparelho autoanalisador BT 3000 plus da marca Wiener $L a b$; (c) os reagentes utilizados para análise foram do mesmo lote (LABORLAB) e os métodos utilizados foram o método enzimático para o colesterol total $(\mathrm{CT})$ e triglicerídeos (TG) e colorimétrico para o HDL-colesterol (HDL-c). O LDL-colesterol (LDL-c) foi calculado por meio da equação de Friedewald. ${ }^{20}$

Os resultados foram analisados com o auxílio do programa SAS® (versão 9.0). Inicialmente realizou-se uma análise exploratória dos dados, descritos em termos de média e desvio padrão para as variáveis quantitativas e em termos de frequência e porcentagem para as variáveis qualitativas. Os dados apresentaram distribuição normal e, desta forma, foram usados coeficientes de correlação de Pearson para verificar as possíveis associações entre as variáveis quantitativas e as comparações estatísticas entre os grupos, tendo como variáveis independentes a classificação pelo IAFG e pelo NHAF foram realizadas por meio de um modelo linear generalizado, considerando sexo e idade como potenciais fatores de confundimento para todas as variáveis, e ainda, cor da pele, para a PAS e PAD. As variáveis confundidoras foram identificadas por meio de análise descritiva dos dados, por estarem distribuídas desigualmente entre os grupos comparados e existirem evidências do ponto de vista biológico. De acordo com a literatura, a divisão dos grupos pelo IAFG deve ocorrer em 
quintis ${ }^{13,17,18}$, entretanto devido à baixa frequência de participantes no grupo "muito fraco", com IAFG de 0 a 99 pontos, e seguindo a recomendação de um mínimo de $10 \%$ do total de participantes em cada grupo para realizar a análise estatística de forma adequa$\mathrm{da}^{21}$, optou-se por unir o grupo "muito fraco" (pontuação 0 a 99) com o grupo "fraco" (pontuação 100 a 199), permanecendo a denominação "fraco" na apresentação dos resultados. O mesmo aconteceu quanto à classificação pelo NHAF, que apresentou uma baixa frequência no grupo "sedentário", o que justificou unir este grupo com o grupo "irregularmente ativo B", sendo denominado desta forma de grupo "sedentário e irregularmente ativo B". Desta forma os indivíduos foram classificados em:

- Grupos IAFG: "fraco" - 0 a 199, "regular" - 200 a 299, "bom" - 300 a 399 e "muito bom" - 400 a 500;

- Grupos NHAF: "sedentário e irregularmente ativo B", "irregularmente ativo A", "ativo" e "muito ativo".

A associação entre os grupos subdivididos pelo IAFG e pelo tempo de prática de exercícios físicos e a supervisão de um profissional de educação física foram avaliadas pelo teste exato de Fischer, sendo que este último seguiu a seguinte categorização:

- Grupos tempo de prática e a supervisão de um profissional de educação física: "grupo não ativo ou com frequência inferior a seis meses de prática de exercícios", "grupo ativo com frequência superior a seis meses de prática de exercícios sem supervisão" e "grupo ativo com frequência superior a seis meses em um programa de exercícios com supervisão".

Este estudo foi aprovado pelo Comitê de Ética em Pesquisa da Faculdade de Ciências Farmacêuticas de Ribeirão Preto da Universidade de São Paulo (CEP/FCFRP n $\left.{ }^{\circ} 172 / 2010\right)$ de acordo com as disposições da Declaração de Helsinque. Todos os indivíduos assinaram o termo de consentimento livre e esclarecido.

\section{Resultados}

A Tabela 1 apresenta as características sociodemográficas e relacionadas à aptidão física (qualitativas) dos participantes.

Tabela 1: Características sociodemográficas e relacionadas à aptidão física (qualitativas) dos participantes

\begin{tabular}{lrc}
\hline Variáveis & $(\mathbf{n}=\mathbf{2 1 3})$ & $\%$ \\
\hline Sexo & 163 & 76,5 \\
Feminino & 50 & 23,5 \\
Masculino & & \\
Cor da Pele & 156 & 73,2 \\
$\quad$ Branca & 57 & 26,8 \\
Parda/Negra & & \\
Faixa Etária & 101 & 47,4 \\
Entre 50 e 59 anos & 112 & 52,6 \\
Entre 60 e 80 anos & & \\
Classificação NHAF & 63 & 29,6 \\
Sedentário e Irregularmente Ativo B & 61 & 28,6 \\
Irregularmente Ativo A & 68 & 31,9 \\
Ativo & 21 & 9,9 \\
Muito Ativo & & \\
Classificação IAFG (pontos) & 61 & 28,6 \\
Fraco (0 a 199) & 58 & 27,2 \\
Regular (200 a 299) & 67 & 31,5 \\
Bom (300 a 399) & 27 & 12,7 \\
Muito bom (400 a 500) &
\end{tabular}

NHAF - nível habitual de atividade física; IAFG - índice de aptidão funcional geral. 
É possível observar equilíbrio entre adultos e idosos, com maior proporção de participantes de cor da pele branca e sexo feminino. Sobre a classificação quanto ao NHAF e IAFG, observa-se menor proporção de participantes nos extremos superiores da ordem de classificação, neste caso, "muito ativo" quanto ao NHAF e "muito bom" quanto ao IAFG. Nos outros grupos, a distribuição acontece, em geral, de forma homogênea.

A Tabela 2 apresenta os resultados obtidos pelo modelo linear generalizado (comparação de grupos) tendo a classificação pelo NHAF como variável independente. Foi possível evidenciar diferença estatística do grupo "muito ativo", "ativo" e "irregularmente ativo A" em comparação ao grupo "sedentário e irregularmente ativo B" para o IMC e, diferença entre o grupo "muito ativo" e "sedentário e irregularmente ativo B" para a PAD.

A Tabela 3 apresenta os resultados do modelo linear generalizado, tendo como variável independente o IAFG. Foi possível evidenciar diferença estatística entre o grupo "muito bom" com "bom", "regular" e "fraco" e, "bom" com "regular" e "fraco" para a variável IMC. Ainda em relação ao IMC, também foi possível evidenciar diferença entre "regular" e "fraco". Sobre o perfil lipídico, os grupos "muito bom" e "bom" apresentaram diferença com "regular" e "fraco" para TG e, "bom" com "fraco" para HDL-c. Em relação à PA, foi possível evidenciar diferença de "muito bom" com "regular" e "fraco" e, "bom" com "fraco" para PAS e PAD, e ainda, de "regular" com "fraco" para PAD.

Como as diferenças entre os grupos nas variáveis apresentadas nas Tabelas 2 e 3 ocorreram em maior quantidade quando os grupos foram subdivididos pelo IAFG, optou-se por utilizar somente esta divisão para a próxima análise. Sendo assim, a Tabela 4 apresenta o resultado do teste exato de Fischer realizado para observar a associação entre os grupos subdivididos pelo IAFG e, os grupos subdivididos pelo tempo de prática de exercícios físicos e a supervisão de um profissional de educação física. Pode-se observar que os grupos "fraco" e "regular" apresentaram maior proporção de indivíduos não ativos ou com prática de exercício físico regular inferior a seis meses. Já nos grupos de IAFG classificado como "bom" e "muito bom" observou-se maior proporção de indivíduos ativos com tempo de prática de exercícios físicos superior a seis meses, com destaque para um maior número de indivíduos realizando a prática com supervisão de profissional de educação física $(\mathrm{p}<0,0001)$.

Tabela 2: Avaliação da aptidão física e do perfil de risco de doenças cardiovasculares em adultos e idosos subdividida de acordo com o nível habitual de atividade física (NHAF)

\begin{tabular}{|c|c|c|c|c|}
\hline & \multicolumn{4}{|c|}{ NHAF } \\
\hline & $\begin{array}{l}\text { Sedent e irreg } \\
\text { atv } B(n=63)\end{array}$ & $\begin{array}{l}\text { Irreg atv } A \\
\quad(n=61)\end{array}$ & $\begin{array}{c}\text { Ativo } \\
(n=68)\end{array}$ & $\begin{array}{l}\text { Muito Ativo } \\
\quad(n=21)\end{array}$ \\
\hline \multicolumn{5}{|l|}{ Avaliação Antropométrica } \\
\hline IMC (kg/m²) & $29,8(5,4)$ & $28(5)^{*}$ & $27,4(4,2)^{*}$ & $27,2(4,2)^{*}$ \\
\hline \multicolumn{5}{|l|}{ Perfil Lipídico } \\
\hline Colesterol Total (mg/dL) & $192,6(35,8)$ & $186,5(34,2)$ & $180(40)$ & $181,7(37,7)$ \\
\hline LDL-colesterol (mg/dL) & $121,4(32,1)$ & $116,8(27,3)$ & $112,6(32,6)$ & $116,2(28,8)$ \\
\hline HDL-colesterol (mg/dL) & $45,8(10,3)$ & $45,7(11,1)$ & $47(9,9)$ & $43,9(9)$ \\
\hline Triglicerídeos (mg/dL) & $131,6(78,9)$ & $111,5(63,9)$ & $105,3(57,1)$ & $108,1(53,2)$ \\
\hline \multicolumn{5}{|l|}{ Pressão Arterial } \\
\hline PAS (mmHg) & $122,9(13,6)$ & $122(14,6)$ & $123(11,4)$ & $119,4(15,1)$ \\
\hline PAD $(\mathrm{mmHg})$ & $81,1(10)$ & $78,4(9,3)$ & $79,2(8,7)$ & $75,1(11,3)^{*}$ \\
\hline
\end{tabular}

Valores expressos em Média (DP); Sedent e irreg atv B - sedentário e irregularmente ativo B; Irreg atv A - irregularmente ativo A; ativo; muito ativo; IMC - índice de massa corporal; PAS - pressão arterial sistólica; PAD - pressão arterial diastólica; ${ }^{*} p<0,05$ versus sedent $e$ irreg atv B; controle das possíveis variáveis geradoras de confusão, idade e sexo, para todas as variáveis, e, para a PAS e PAD idade, sexo e cor da pele. 
Tabela 3: Avaliação da aptidão física e do perfil de risco de doenças cardiovasculares em idosos subdividida de acordo com o Índice de Aptidão Funcional Geral (IAFG)

\begin{tabular}{|c|c|c|c|c|}
\hline & \multicolumn{4}{|c|}{ IAFG } \\
\hline & Fraco $(n=61)$ & Regular $(n=58)$ & Bom $(n=67)$ & Muito Bom $(n=27)$ \\
\hline \multicolumn{5}{|l|}{ Avaliação Antropométrica } \\
\hline IMC $\left(\mathrm{kg} / \mathrm{m}^{2}\right)$ & $30,7(5,1)$ & $28,9(5,1)^{*}$ & $26,9(3,7)^{*} \dagger$ & $24,9(3,3)^{\star \star \ddagger}$ \\
\hline \multicolumn{5}{|l|}{ Perfil Lipídico } \\
\hline Colesterol Total (mg/dL) & $190,4(38,7)$ & $188,6(36,3)$ & $181,3(39)$ & $180,3(28,8)$ \\
\hline LDL-colesterol (mg/dL) & $120(35)$ & $116,9(29,3)$ & $114(31,2)$ & $115,9(20,3)$ \\
\hline HDL-colesterol (mg/dL) & $44,3(10,7)$ & $46,1(9,4)$ & $47,6(10,8)^{*}$ & $45,3(9,5)$ \\
\hline Triglicerídeos (mg/dL) & $127,2(73,9)$ & $123,50(65,5)$ & $104(64)^{*} \dagger$ & $97,4(48)^{*} \dagger$ \\
\hline \multicolumn{5}{|l|}{ Pressão Arterial } \\
\hline PAS $(\mathrm{mmHg})$ & $127,1(13,6)$ & $123,3(12,9)$ & $120,1(11,3)^{*}$ & $115,1(14,7)^{* \dagger}$ \\
\hline PAD (mmHg) & $82,9(9,7)$ & $79,7(9,4)^{*}$ & $77,2(8,2)^{*}$ & $74,3(10,3)^{*} \dagger$ \\
\hline
\end{tabular}

Valores expressos em Média (DP); Pontuação na Bateria de Testes: Fraco: 0 a 199; Regular: 200 a 299; Bom: 300 a 399; Muito bom: 400 a 500. IMC - índice de massa corporal; PAS - pressão arterial sistólica; PAD - pressão arterial diastólica; ${ }^{*} p<0,05$ versus Fraco; ${ }^{\dagger} p<0,05$ versus Regular; ${ }^{\ddagger} p<0,05$ versus Bom; controle das possíveis variáveis geradoras de confusão, idade e sexo, para todas as variáveis, e, para a PAS e PAD idade, sexo e cor da pele.

Tabela 4: Distribuição dos participantes quanto ao tempo de prática de exercícios físicos e a supervisão de um profissional de educação física nos grupos tendo o Índice de Aptidão Funcional Geral (IAFG) como variável independente

\begin{tabular}{|c|c|c|c|c|c|c|c|c|}
\hline & \multicolumn{8}{|c|}{ IAFG } \\
\hline & \multicolumn{2}{|c|}{ Fraco } & \multicolumn{2}{|c|}{ Regular } & \multicolumn{2}{|c|}{ Bom } & \multicolumn{2}{|c|}{ Muito Bom } \\
\hline & $\mathrm{n}$ & $\%$ & $\mathrm{n}$ & $\%$ & $\mathrm{n}$ & $\%$ & $\mathrm{n}$ & $\%$ \\
\hline \multicolumn{9}{|l|}{ Tempo de Prática/Supervisão } \\
\hline$<6$ meses & 45 & 73,8 & 45 & 77,6 & 14 & 20,9 & 4 & 14,8 \\
\hline > 6 meses sem supervisão & 15 & 24,6 & 7 & 12,1 & 18 & 26,9 & 4 & 14,8 \\
\hline > 6 meses com supervisão & 1 & 1,6 & 6 & 10,3 & 35 & 52,2 & 19 & 70,4 \\
\hline Total & 61 & 100 & 58 & 100 & 67 & 100 & 27 & 100 \\
\hline
\end{tabular}

Associação estatística $p<0,0001 ;<6$ meses - grupo não ativo ou com frequência inferior a seis meses de prática de exercícios; $>6$ meses sem supervisão - grupo ativo com frequência superior a seis meses de prática de exercícios sem supervisão e; > 6 meses com supervisão - grupo ativo com frequência superior a seis meses em um programa de exercícios com supervisão.

\section{Discussão}

Com o objetivo de comparar a relação do NHAF e da aptidão física com a saúde cardiovascular de adultos e idosos, os resultados deste estudo sugerem que as variáveis relacionadas à saúde cardiovascular pos- suem maior associação com o IAFG quando comparada com o NHAF neste segmento populacional.

Em termos de caracterização dos participantes, a amostra do presente estudo apresentou composição em sua maioria por mulheres e cor da pele branca. A maioria dos estudos com esta faixa etária apresenta 
predominância de participantes do sexo feminino. ${ }^{11,13,22,23}$ A justificativa apontada para este fato é que os homens se envolvem menos em programas sociais e comunitários e possuem uma rotina menos evidente de busca pela saúde. ${ }^{24}$ Com relação à cor da pele, apesar da diferença apresentada, nas análises em que poderiam oferecer alguma influência (PAS e PAD), a cor da pele foi controlada como variável geradora de confusão.

Ainda em relação aos dados da Tabela 1, os resultados do NHAF, se aproximam dos resultados do estudo de base populacional em Ribeirão Preto/SP que apontaram para a prática suficiente de atividade física (ativos e muito ativos) em aproximadamente $40 \%$ dos homens e $32 \%$ das mulheres, levando em consideração apenas os indivíduos com idade superior a 50 anos. ${ }^{25} \mathrm{O}$ presente estudo, incluindo homens e mulheres, apresentou prática suficiente de atividade física de 41,8\%. Quanto aos resultados do IAFG, a frequência em cada grupo também se aproxima da frequência apresentada por outros estudos. ${ }^{13,17,18}$

Se analisarmos tanto o NHAF quanto o IAFG, ambos possuem percentuais parecidos em termos de classificação, ou seja, 58,2\% dos participantes foram classificados como sedentários ou irregularmente ativos para o NHAF e, 55,8\% como fraco ou regular para o IAFG. De certa forma esses dados mostram certa homogeneidade para ambas as avaliações. No entanto, as relações que ambas possuem com o perfil de risco para as doenças cardiovasculares são diferentes.

Quando os participantes foram subdivididos de acordo com o NHAF (Tabela 2), o modelo linear generalizado somente detectou diferença entre alguns grupos para o IMC e PAD. A literatura apresenta alguns estudos com resultados na mesma direção, como exemplo, Gonçalves e cols. ${ }^{26}$, que encontraram associação entre IMC e NHAF em adultos e idosos: os indivíduos classificados como muito ativos estavam no peso ideal, já os irregularmente ativos e sedentários, em sua maioria, apresentaram excesso de peso. Por outro lado, outros estudos não apresentaram associações entre o NHAF e IMC, como é o caso do estudo de Michelin e cols. ${ }^{10}$, realizado com adultos e idosos, no qual houve predomínio de NHAF moderado e alto nos grupos com excesso de peso e adiposidade abdominal. Os autores apontaram para a necessidade de ampliação da reflexão na relação entre exercício físico e saúde, já que os resultados sugeriram que o NHAF pareceu ser insuficiente na manutenção da composição corporal.
Com relação a PA, o presente estudo encontrou uma associação entre NHAF e PAD, no entanto, outros estudos, por exemplo, Zaitune e cols. ${ }^{11}$, não encontraram associações entre ambas as variáveis.

Em termos gerais, o NHAF não possui fortes associações com os fatores de risco de DCVs. Corroborando esta afirmação, um estudo realizado com homens e mulheres idosos não encontrou diferenças entre os grupos classificados pelo NHAF para as variáveis IMC, circunferência da cintura, glicemia, TG, HDL-c, PAS e PAD. ${ }^{12}$

Considerando a importância da atividade física para a saúde, o desenvolvimento de ferramentas para avaliação do NHAF foi essencial. No entanto, até meados de 1990, a comparação de dados sobre atividade física coletados em diversos estudos nas diferentes partes do mundo ficou limitada devido ao uso de instrumentos diferentes. Para superar esta limitação, um grupo de pesquisadores de vários países propôs um questionário padronizado, culturalmente adaptável para medir o NHAF em nível populacional: o IPAQ. ${ }^{27}$ Este instrumento, amplamente utilizado para a avaliação e monitoramento do NHAF nas populações latino-americanas, oferece ampla variedade de dados, mas apresenta algumas limitações, principalmente relacionada à subjetividade das respostas do participante e, consequentemente, no estabelecimento de relações com os fatores de risco para DCVs. Portanto, a percepção subjetiva do NHAF pode não estar refletindo a real condição da aptidão física e saúde cardiovascular do indivíduo. Apenas a título de exemplificação, uma pessoa que realiza a prática da caminhada todos os dias da semana por pelo menos 30 minutos, terá uma percepção do NHAF de ativo fisicamente. No entanto, se esta caminhada estiver sendo realizada com baixa intensidade, é possível que os benefícios para a saúde cardiovascular provenientes desta prática sejam mínimos. Este aspecto é discutido no estudo de Trapé e cols. ${ }^{28}$ que apontou para benefícios limitados à saúde cardiovascular no grupo de adultos e idosos que realizavam caminhada não supervisionada.

Sobre as associações encontradas entre IAFG e as variáveis relacionadas à saúde cardiovascular (Tabela 3), observou-se diferença nos grupos quanto ao IAFG para o IMC, HDL-c, TG, PAS e PAD, sendo que os grupos com IAFG mais elevados apresentaram melhores resultados em todas estas variáveis. Estes resultados sugerem uma relação mais forte entre o IAFG e as variáveis relacionadas aos fatores de 
risco para DCVs do que a relação entre o NHAF e os mesmos fatores, mostrando que o IAFG, obtido por uma bateria de testes, pode ser uma alternativa mais coerente quando se almeja estabelecer tais relações.

Cabe a ressalva que não é o escopo deste trabalho desvalorizar tanto o instrumento quanto as pesquisas que utilizaram o questionário IPAQ para avaliação do NHAF, mas sim, apontar que é um instrumento que possui limitações. O IPAQ continua sendo importante no levantamento de dados, entretanto, o IAFG se mostrou mais eficiente para estabelecer uma relação entre aptidão física e saúde cardiovascular.

Diante deste fato, é importante destacar a importância de um olhar mais amplo para a relação entre e saúde cardiovascular e estilo de vida ativo, chamando a atenção deste último, para o melhor desenvolvimento da aptidão física e sugerindo que estudos futuros utilizem o treinamento multicomponente como forma de intervenção.

Por muitos anos a discussão acerca dos benefícios do exercício físico para a saúde ficou restrita à realização de exercícios aeróbicos. ${ }^{7,29,30}$ Entretanto, ao longo dos últimos anos, o treinamento de força e outros tipos de exercícios que envolvem a flexibilidade e equilíbrio vêm sendo recomendados como importantes componentes nos programas de exercícios físicos para adultos e idosos., ${ }^{5,31,32}$ Isto vem acontecendo, provavelmente, pelo conhecimento de que o processo de envelhecimento acomete as diversas capacidades e habilidades motoras e que cada modalidade de exercício físico apresenta benefícios específicos. Logo, pensar em uma intervenção com um único tipo de exercício físico, pode não ser o mais adequado. Como exemplo, temos estabelecido na literatura que o exercício de força apresenta mais benefícios para a saúde óssea e muscular ${ }^{5}$, enquanto o treinamento aeróbico é mais indicado para controle da HAS, perfil lipídico, dentre outros. ${ }^{5,6}$ Por isto, apesar de termos poucas evidências de estudos que buscaram estabelecer relações entre aptidão física e saúde cardiovascular ${ }^{33}$ ou intervenções que buscaram verificar o efeito de um programa de exercícios físicos multicomponentes na saúde cardiovascular ${ }^{23}$, estudos recentes que realizaram intervenções comparando os efeitos do treinamento aeróbico e de força em variáveis relacionadas à saúde cardiovascular, têm discutido a ideia de pensar de forma mais ampla a relação entre estilo de vida ativo e saúde cardiovascular, sugerindo intervenções que busquem verificar a melhor relação entre aptidão física e a saúde cardiovascular, além do efeito do treinamento multicomponente propriamente dito. ${ }^{34,35}$ Vale ressaltar que os posicionamentos oficiais relacionados aos fatores de risco apresentados ${ }^{5,7,29,30,31,32}$ estabelecem um olhar mais amplo nos programas de exercícios físicos voltados para a saúde cardiovascular, incluindo treinamento aeróbico, de força, alongamentos, entre outros aspectos. Na Tabela 4 observou-se a associação entre o nível de aptidão física dos participantes e o tempo de prática de exercícios físicos e a supervisão de um profissional de educação física. Desta forma, é possível fazer uma relação direta entre a definição de exercício físico apresentada no início deste estudo e os resultados encontrados, já que os grupos com melhores classificações do IAFG "bom" e "muito bom" apresentaram maior proporção de indivíduos ativos ou com prática superior a seis meses de exercícios, destacando-se dentre eles os com prática supervisionada por um profissional de educação física.

Sobre os resultados melhores nos grupos com prática de exercícios físicos superior a seis meses (Tabela 4), vale ressaltar que a prática de exercícios físicos deve ser regular para alcançar os benefícios para a saúde e manutenção dos níveis de aptidão física, como bem apontado pelos posicionamentos oficiais sobre prescrição de exercícios físicos para adultos e idosos. ${ }^{5,31,32}$

Em relação ao que foi encontrado na literatura a respeito da prática supervisionada de exercícios físicos, alguns estudos destacam a figura do profissional de educação física. A "Diretriz de Reabilitação Cardíaca" ${ }^{36}$ afirmou que o treinamento físico na intervenção primária e secundária de DCVs é custo/efetivo, desde que seja considerada uma adesão adequada ao programa, sendo que esta adesão pode ter relação com o acompanhamento supervisionado, principalmente entre mulheres de 55 a 74 anos. Interessante notar que a presença de um profissional qualificado influenciou a adesão dos participantes em um programa de exercícios físicos, ressaltando que a presença de um profissional qualificado é extremamente importante tanto para a adesão quanto para os resultados esperados com esta prática. ${ }^{37} \mathrm{E}$, no estudo de Trapé e cols. ${ }^{28}$, os grupos de adultos e idosos que realizavam uma ou duas modalidades de exercícios físicos supervisionados por um profissional de educação física, além da caminhada não supervisionada, apresentaram melhores resultados nas variáveis relacionadas à aptidão física e saúde cardiovascular quando comparados ao grupo que realizava apenas a caminhada não supervisionada. 


\section{Conclusão}

Os resultados do presente estudo sugerem que existe maior evidência de associação entre saúde cardiovascular e a aptidão física do que com o NHAF, além de maior proporção de indivíduos com prática regular de exercício físicos superior a seis meses e com supervisão de um profissional de educação física, nos grupos de aptidão física mais elevada. Assim, a avaliação direta da aptidão física parece ser mais indicada quando se almeja relacionar com a saúde cardiovascular. Desta forma, sugerem-se políticas públicas com olhar mais global para o planejamento de programas relacionados à prática de exercícios físicos, priorizando a melhora da aptidão física propriamente dita e destacando a figura do profissional de educação física como orientador de uma prática correta e segura, de modo a maximizar os benefícios da prática de exercícios físicos.

\section{Agradecimentos: CNPQ 557967/2009-0 e FAPESP 09/54586-0}

\section{Referências}

1. Mansur AP, Lopes AIA, Favarato D, Avakian SD, César LAM, Ramires JAF. Transição epidemiológica da mortalidade por doenças circulatórias no Brasil. Arq Bras Cardiol. 2009; 93:506-10.

2. Mansur AP, Favarato D. Mortalidade por doenças cardiovasculares no Brasil e na região metropolitana de São Paulo: atualização 2011. Arq Bras Cardiol. 2012;99:755-61.

3. Papaléo Neto M. Autonomia e independência. In: Papaléo Neto $M$, editor. Gerontologia: a velhice e o envelhecimento em visão globalizada. São Paulo: Atheneu; 1996. p. 313-30.

4. Carpensen CJ, Powel KE, Christenson, GM. Physical activity, exercise, and physical fitness: definitions and distinctions for health-related research. Public Health Rep. 1985;100:126-31.

5. Chodzko-Zajko WJ, Proctor DN, Singh MA, Minson CT, Nigg CR, Salem GJ, et al. American College of Sports Medicine position stand: exercise and physical activity for older adults. Med Sci Sports Exerc. 2009;41:1510-30.

6. Pitanga FJG. Epidemiologia da atividade física, do exercício e da saúde. 3ª edição. São Paulo: Phorte; 2010.

7. Sociedade Brasileira de Cardiologia/Sociedade Brasileira de Hipertensão/Sociedade Brasileira de Nefrologia. VI Diretrizes Brasileiras de Hipertensão. Arq Bras Cardiol. 2010;95(1 supl 1): 1-51.

8. BRASIL. Ministério da Saúde, Secretaria de Vigilância em Saúde, Vigilância de Fatores de Risco e Proteção Para Doenças Crônicas por Inquérito Telefônico (VIGITEL). Vigilância de fatores de Risco e proteção para doenças crônicas por inquérito telefônico - Brasil 2011. Brasília: Ministério da Saúde, 2012.
9. Hallal PC, Andersen LB, Bull FC, Guthold R, Haskell W. Global physical activity levels: surveillance progress, pitfalls and prospects. Lancet. 2012;380(9838):247-57.

10. Michelin E, Corrente JE, Burini, RC. Associação dos níveis de atividade física com indicadores socioeconômicos, de obesidade e de aptidão física em adultos. Rev Bras Ativ Fis Saude. 2010;15:35-41.

11. Zaitune MPA, Barros MBA, Galvão César CL, Carandina L, Goldsbaum M. Hipertensão arterial em idosos: prevalência, fatores associados e práticas de controle no Município de Campinas. Cad Saude Publica. 2006;22:285-94.

12. Dalacorte RR. Síndrome metabólica e atividade física em idosos de uma comunidade do sul do Brasil [Dissertação de Mestrado]. Ribeirão Preto: Universidade de São Paulo; 2008.

13. Zago AS, Gobbi S. Valores normativos da aptidão funcional de mulheres de 60 a 70 anos. Rev Bras Cienc Mov. 2003; 11:77-86.

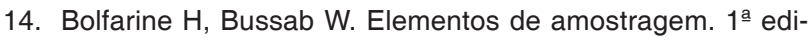
ção. São Paulo: Edgard Blucher; 2005.

15. Matsudo S, Araujo T, Matsudo V, Andrade D, Oliveira LC, Braggionn A. Questionário Internacional de Atividade Física (IPAQ): estudo de validade e reprodutibilidade no Brasil. Rev Bras Ativ Fis Saude. 2001; 6:5-18.

16. Rikli RE, Jones CJ. Development and validation of a functional fitness test for community-residing older adults. J Aging Phys Act. 1999;7:129-81.

17. Benedetti TRB, Mazo GZ, Gobbi S, Amorim M, Gobbi LTB, Ferreira L. Valores normativos de aptidão funcional em muIheres de 70 a 79 anos. Rev Bras Cineantropom Desempenho Hum. 2007;9:28-36.

18. Mazo GZ, Benedetti TRB, Gobbi S, Ferreira L, Lopes MA. Valores normativos e aptidão funcional em homens de 60 a 69 anos. Rev Bras Cineantropom Desempenho Hum. 2010;12:316-23.

19. Organização Mundial da Saúde. Obesity: preventing and managing the global epidemic. Geneva: WHO Technical Report Series 894; 2000 .

20. Warnick GR, Knopp RH, Fitzpatrick V, Branson L. Estimating low-density lipoprotein cholesterol by the Friedewald eqation is an adequate for classifying patients on the basis of nationally recommended cutpoints. Clin Chem. 1990;36:15-29.

21. Hollander M, Wolf DA. Nonparametric statistical method. New York: John Wiley \& Sons; 1999.

22. Andreotti MC, Okuma SS. Perfil sócio-demográfico e de adesão inicial de idosos ingressantes em um programa de educação física. Rev Paul Educ Fis. 2003;17:142-53.

23. Marques E, Carvalho J, Soares JMC, Marques F, Mota J. Effects of resistance and multicomponent exercise on lipid profiles of older women. Maturitas. 2009;63:84-8.

24. Gomes R, Nascimento EF, Araujo FC. Por que os homens buscam menos os serviços de saúde do que as mulheres? As explicações de homens com baixa escolaridade e homens com ensino superior. Cad Saude Publica. 2007;23:565-74.

25. Suzuki CS, Moraes AS, Freitas ICM. Atividade física e fatores associados em adultos residentes em Ribeirão Preto, SP. Rev Saúde Publica. 2011;45:311-20.

26. Gonçalves HR, Gonçalves LAP, Costa TA, Goulart MI. Associação entre IMC e atividade física em adultos de diferentes níveis socioeconômicos. Revista Saúde e Pesquisa. 2011;4:161-8. 
27. Hallal PC, Gomez LF, Parra DC, Lobelo F, Mosquera J, Florindo $A A$, et al. Lições aprendidas depois de 10 anos de uso do IPAQ no Brasil e na Colômbia. J Phys Act Health. 2010;7(2 supl 2):S259-64.

28. Trape AA, Sacardo AL, Cássia AF, Monteiro HL, Zago AS. Relação entre a prática da caminhada não supervisionada e fatores de risco para as doenças cardiovasculares em adultos e idosos. Medicina (Ribeirão Preto). 2014;47:165-76.

29. Donelly JE, Blair SN, Jakicic JM, Manore MM, Rankin JW, Smith BK. American College of Sports Medicine Positions Stand. Appropriate physical activity intervention strategies for weight loss and prevention of weight regain for adults. Med Sci Sports Exerc. 2009;41:459-71.

30. Xavier HT, Izar MC, Faria Neto JR, Assad MH, Rocha VZ, Sposito AC, et al. Sociedade Brasileira de Cardiologia. V Diretriz de Dislipidemias e Prevenção da Aterosclerose. Arq Bras Cardiol. 2013;101(4 Supl. 1):1-32

31. Garber CE, Blissmer B, Deschenes MR, Franklin BA, Lamonte MJ, Lee I, et al. Quantity and quality of exercise for developing and maintaining cardiorespiratory, musculoskeletal, and neuromotor fitness in apparently healthy adults: guidance for prescribing exercise. Med Sci Sports Exerc. 2011; 43:1334-59.
32. Haskell WL, Lee I, Pate RR, Powell KE, Blair SN, Franklin, BA, et al. Physical activity and public health: updated recommendations for adults from the American College of Sports Medicine and the American Heart Association. Med Sci Sports Exerc. 2007;39:1423-34.

33. Mazo GZ, Kulkamp W, Lyra VB, Prado APM. Aptidão functional geral e IMC de idosas praticantes de atividade física. Rev Bras Cineantropom Desempenho Hum. 2006;8:46-51.

34. Collier SR, Frechette V, Sandberg K, Schafer P, Ji H, Smulyan $\mathrm{H}$, et al. Sex differences in resting hemodynamics and arterial stiffness following 4 weeks of resistance versus aerobic exercise training in individuals with pre-hypertension to stage 1 hypertension. Biol Sex Differ. 2011;2:1-7.

35. Fett CA, Fett WCR, Marchini JS. Exercício resistido vs Jogging em fatores de risco metabólicos de mulheres com sobrepeso/obesas. Arq Bras Cardiol. 2009;93:519-25.

36. Sociedade Brasileira de Cardiologia. Diretriz de reabilitação cardíaca. Arq Bras Cardiol. 2005;84:431-40.

37. Cabral-de-Oliveira AC, Ramos PS, Araújo CGS. Distância do domicílio ao local de exercício físico não Influenciou a aderência de 796 participantes. Arq Bras Cardiol. 2012; 98:553-8. 